removed the greater part of it from the very posterior part of the canal, from a pouch, in fact, at the apex of the prostate, if not, indeed, in the substance of the gland itself. You will observe that means are here afforded for reducing the size of the fragment, if it does not come readily along the canal; but if a foreign body passes the orifice of the bladder, and can be seized as $I$ have described, there will seldom be great difficulty in bringing it along to the very external orifice of the urethra, and from that it can be disentangled by a very slight dilatation.

The operation of lithotrity may now be looked upon as tolerably perfect; and if proper cases be selected, it is likely to be very successful in its results, and to be much less dangerous than even lithotomy performed in the best manner and by experienced operators. It certainly is not likely to be attended with the same risks and the same dreadful mortality that attended upon it in the first few years after its introduction. We then heard of the instruments breaking in the bladder, and, what was worse, of their bending in such a way that they could not again be extracted from the viscus. Then came all sorts of incisions and attempts to twist back the blades of the instrument. I need not tell you that many patients who, if subjected to lithotomy, would probably have recovered without accident, were sacrificed to these insane attempts by incompetent men, with insufficient means. Now no such dread need be entertained, as apparatus in every way suited for the purpose is to be had. The operation, I may say, in conclusion, is not well adapted to young subjects. The parts in them are excessively irritable, and the stones are, in the majority of cases, excessively hard. The fragments are got rid of with difficulty, and altogether $I$ firmly believe that the operation of lithotomy is much to be preferred for young patients. I veuture to express this opinion after having had ample experience. The operation of cutting children for stone, I may add, is unattended, in the hands of an experienced and expert surgeon, with risk of any kind, and, indeed, the mortality is not, or should not be, worth noticing; say one death in some hundreds of cases.

\section{PRODUCTION OF PREMATURE LABOUR.}

To the Editor of The Lancet.

Sir,-If the following reports are of sufficient interest, you will much oblige me by inserting them in your widely-circulated Journal. I remain, Sir, your most obedient servant,

R. Forester Wells, M.R.C.S. Steward.street, Bishopsgate Without, Oct. 18, 1843 .
Case 1.-Mrs. R., a delicate woman, of spare habit and short stature, was taken in labour with her first child on Friday evening, March 29, 1839, and on Sunday molning the liquor amnii escaped. On Monday, at nine o'clock, a.m., I was requested to see her under the following circumstances:The uterus was acting violently every three minutes, its mouth almost completely dilated, and with the vagina very much swollen and excessively tender; the child's head in its natural position, fixed between the promontory of the sacrum and the pubis, and I was informed that it had been so for the previous twelve hours; the pulse was full and quick; much pain in the head; the tongue coated with a white fur, and the patient in a ver'y restless state; the bowels had recently acted, but no water had passed for twelve hours. Six. teen ounces of blood, very buffy, were drawn from the arm, and the water was drawn off to the extent of ten ounces.

At two o'clock, p.m., the child's head being in the same situation, I attempted, but unsuccessfully, to apply the long forceps; but after two hours and a half labour succeeded in delivering her with the perforator and craniotomy forceps. I then ascertained that the antero-posterior diameter of the pelvis was below three inches. She had a favourable recovery.

On the 13th of August, 1840, I was called to attend the same patient in labour at the seventh month; there was alarming hæmorrhage; the feet presented, and the os uteri was perfectly dilated. I ruptured the mem. branes,-the hæmorthage abated, and I succeeded in delivering the child's body in about half an hour, but was two hours in getting the head through the brim of the pelvis. The child was dead, the placenta adhered, and an unusual quantity of blood followed it. She had a very protracted recovery, I fully confirmed my previously-formed opinion of the deficiency in the dimensions of the pelvis. I then recommended the induction of premature labour at the seventh month in the event of another pregnancy, and on the 20 th of $\mathrm{De}$. cember, 1841, precisely seven months from the termination of her last menstruation, I commenced at twelve o'clock, noon, giving her the mixture recommended by Dr. Ramsbotham (Obstetric Medicine and Surgery, p. 379 ); at the same time with the index finger of the left hand I slightly separated the membranes from the os and cervix uteri, and ascertained the presenting part to be the head. I repeated this operation at the same hour the following day, and on Wed. nesday, the os uteri being about one inch in diameter, soft and dilatable, I ruptured the membranes. From this time labour was fairly established and proceeded in the usual way; and at ten o'clock on Thursday evening, eighty-two hours from the commencement of the use of the medicine, and thirty. 
four after the rupture of the membranes, she was delivered of a boy, which lived twelve hours. The head passed easily through the pelvis, and the recovery was rapid.

CAse 2. - On the 20th of last month, seven months and a half from the last menstruation, the same patient applied to me again, and a similar practice was adopted, the membranes being ruptured rather earlier ; the head, which presented, passed through the pelvis with considerable difficulty, and she was delivered of a female child, now alive and well, sixty-five hours after the first dose of the medicine, and thirty. eight after the ruptare of the membranes. An inflammation of the breast slightly retarded her recovery.

CASE 3.- Mrs. F., retat. 34, of short stature, and very sanguine temperament, has been married nine years. Her first child was very small, and died a few minutes after its birth ; the labour lasted serenty-two hours. Her second child was dead-born, after a protracted labour. Her third child, premature, at the eighth month; lived to be six years old. During these three labours she was attended by a midwife, and after each had an attack of uterine inflammation; Her fourth was an abortion, of the fifth month.

Her fifth child was dead-born, after a very protracted labour. It was during her sixth labour I was called to see her; a portion of the placenta, with the feet, presented ; the hæmorthage was very alarming, and there was much difficulty in forcing the head through the pelvis. The child was of six months' growth, and was large, considering the period it lived-twelve hours. In this case the pelvis is altogether small, the promontory of the sacrum projects, and the antero-posterior diameter is two inches and three quarters. I then recommended the induction of premature labour in the event of another pregnancy. She, however, did not consent, and on the 18th of March, 1842, after a very laborious labour of forty-eight hour's duration, I delivered her, by craniotomy, of a large male child. Uterine inflammation followed, requiring active antiphlogistic treatment.

On the 2nd of July, 1843, being pregnant for the eighth time, and precisely eight months from the last menstruation, she consented to have labour brought on. The same practice was adopted as in Mrs. R.'s case, and she was delivered of a child (now living) fifty-four hours after the first dose of the medicine, and four after the rupture of the membranes. Very active uterine action commenced immediately after the administration of the medicine, and from my not being able to visit ber sooner the os uteri was completely dilated before the rupture of the membranes.

CASE 4.- Mrs. L., ætat. 24, a short and ill-made woman, after a very severe labour of sixty hours duration, was delivered of a dead child on the 20th of August, 1840, under the care of a midwife. The head, according to her own description, was flattened from side to side, and very much bruised; she recovered slowly.

On the 7th of September, 1841, she was delivered of her second child (dead), under the care of a practitioner of midwifery. The feet presented, and there was considerable difficulty in extracting the head; the child was rather below the average size.

On the 31st of August, 1842, the same patient being in labour of her third child, I was requested to see her twenty-four hours after the natural evacuation of the waters. The os uteri was perfectly dilated; a portion of it, very much swollen, was hanging down, compressed, between the child's head and the brim of the pelvis. The head was immoveably fixed, and it was impossible to introduce a finger between it and the pelvic brim at any part of its circumference. The pains, which had been very violent, had almost entirely subsided; there was great irritability. She had had no sleep for two nights, and complained of pain in the head. I prescribed thirty minims of laudanum, and on visiting her eight hours after, I found she had had some refreshing sleep. The pains were returning, but made no impression on the child's head, which remained in statu quo. After perforating and removing a considerable portion of the cranium, I completed the delivery with the craniotomy forceps. She recovered slowly. The antero-posterior diameter in this case is below three inches.

On the 21st of September, 1343, precisely eight months from her last menstruation, she applied, according to my recommendation, to have premature labour induced. Precisely the same practice was adopted as in the other cases, and she was delivered of a living child, sixty-nine hours after the first dose of medicine, and fourteen after the rupture of the membranes, and, to use her own expression, " never had so good a getting-up."

For the purpose of rupturing the membranes I used the stilette of a male catheter, with the index and second fingers of the left hand as a director. I had no difficulty in ascerlaining the presenting part of the foetus at the first examination in any of the above cases, excepting Mrs. F.'s, where the want of space was so great as to make it difficult to reach the os uteri.

The three methods recommended to be used, separately, by obstetric writers have been conjointly employed in these cases, namely, the separation of the membranes and slight dilatation of the os uteri, the artificial evacuation of the waters, and the administration of the ergot of rye, and, as the result proves, with complete success. Dr. Ramsbotham says he has never found the two last means fail ; and I think that where the first can be accomplished with ease, it enhances 
the probability of success. Dr. Robert Lee, in his " Clinical Midwifery," relates a case in which seven ounces of ergot were taken without producing the desired effect; but that is not sufficient to overthrow the mass of evidence which exists of its efficacy, not only in increasing, but likewise in originating uterine action, and used conjointly with the other means, $\mathbf{I}$ think there is little fear of its producing a deleterious effect on the child.

\section{CAUSE OF COAGULATION OF BLOOD.}

To the Editor.-Sir: In the present uncertainty respecting the coagulation of blood, allow me to ask whether any fact is opposed to the hypothesis that the halitus exhaled from the blood (this exhalation being the first phenomenon that is observed in coagulation) is the agent to which we should attribute the solution of the fibrin in the blood,in a form perhaps somewhat similar to the akaline menstruum which maintains the fluidity of albumen? A heat of $120^{\circ}$, exposure by a broad surface to atmospheric influence and agitation, favour the separation of fibrine. These circumstances, likewise, might be expected to favour the extrication of the hali. tus. Cold, even to freezing, a small surface of exposure, \&c., the very circumstances which lessen the exhalation, restrain the tendency of the fibrine to solidify; whilst a coating of oil effectually prevents both. As an alkaloid substance possessing properties such as $I$ ascribe to the halitus, I would mention phosphuretted hydrogen; without, however, having any reason to suppose that that hyduret is actually the agent concerned. However, phosphuretted hydrogen is found in the blood, combined with a fatty acid. I have the honour, Sir, to remain, your obedient servant,

University College, London, Oct. 17, 1843.

\section{EFFECTS OF TURPENTINE ON} THE DOG.

\section{To the Editor of THE LANCET.}

Sir,-The number of your very valuable publication for August 26th ult. (page 784), contains an article extracted from the "Veterinarian," written by Mr. Mather, on the baneful effects of turpentine on the canine species, a similar case having previously come under my own observation, but ending fatally. In the course of last autumn a gentleman called upon me, who said that he had a very valuable dog; of the terrier breed, which he thought was much infested with worms, and wished to know what I would advise him to administer for their expulsion. I, told him that I was not a veterinary surgeon, but knowing the value of turpentine as a mechanical anthelmintic in the human system, and not being aware that there existed any essential difference in the economy of the dog and that of man, so that turpentine should operate on the former as a virulent poison, and on the latter as a safe and valuable remedy, and also recollecting the dose which may be administered with impunity in the human species, $I$ at once stated that I should recommend turpentine to be given to his dog, in the dose of a small dessert-spoonful, and that, after a few doses, I had no doubt but that he would find some of the worms expelled. On the following day he called upon me, very much displeased at the advice; for immediately after administering the turpentine the dog gave a yell, reeled a few yards from him, and expired as though in a violent paroxysm of convulsions. This case very much strengthens the facts related by Mr. Mather, and confirms his views, that turpentine cannot be administered with the same impunity to the lower animals as to man. I am, Sir, your obedient servant,

R. S. Nightingale, M.R.C.S., Late Surgeon to the Liverpool Eastern October 16, 1843.

$$
\text { Dispensary. }
$$

\section{NEW MEANS OF TESTING BUFFY} BLOOD.

Mr. T. Wharton Jones, in the "Edin. Med. Journ." for October, suggests the following experiments for determining, by a drop, whether blood presents those constituents which would render it " buffy" when drawn in ordinary venesection:-Draw a drop of healthy blood; receive it on a plate of glass; lay a thinner plate over it. Forthwith hold it up against the light, and it will present a uniform aspect, but in half a minute it will be finely mottled. If the blood be buffy, it will, treated in the same way, be more thinly spread out; and the mottle will present itself at once, and more evidently than in healthy blood at the end of a minute, in time increasing still more. Healthy blood is thicker than buffy blood. In buffy blood, the mottled appearance presents itself as soon as the examination can be made, and, more distinctly, from the Jargeness of the clear interspaces. If the healthy blood thus spread out be immediately examined under the microscope, the red corpuscles are observed dispersed confusedly about in the liquor sanguinis. (1) Such is the condition on which depends the uniform appearance first seen by the naked eye. In half a minute, or so, the red corpuscles overlap each other, and then rise up on edge, and become ap. plied side to side. (2) Like coins in rolls, they occupy less space than when irregularly dispersed, with spaces containing nothing but liquor sanguinis. (3) Such is the condition on which the finely mottled appearance depends. 